\title{
Análisis de los efectos que se producen al utilizar repuestos genéricos en el sistema de control de emisiones del Chevrolet Sail 1,4l 2012
}

\section{Analysis of the Effects that Occur When Using Generic Spare Parts in the Emissions Control System Chevrolet Sail 1.41 2012}

MSc. Marco V Noroña M

Universidad Internacional del Ecuador, Ecuador

Autor para correspondencia: manoroname@uide.edu.ec

Fecha de recepción: 22 de Agosto de 2017 - Fecha de aceptación: 30 de Septiembre de 2017

Resumen: El presente artículo tiene como objetivo analizar si utilizando repuestos genéricos en el vehículo Chevrolet Sail 1.4L específicamente el filtro de admisión de aire del motor, las bujías y modificando la calibración de los electrodos de la bujía conseguimos reducir el porcentaje de emisiones de gases contaminantes al medio ambiente. La importancia de controlar las emisiones de los gases contaminantes por el bienestar de los seres humanos y de nuestro planeta. Actualmente la capa de ozono se encuentra deteriorada productos de gases nocivos que provocan el efecto invernadero. La combustión en los motores es la principal causa de contaminación ambiental por lo que se ha implementado la electrónica y demás dispositivos para controlar y reducir la contaminación generada por la combustión en los motores. Se realizaron 192 pruebas de laboratorio donde se utilizaron 4 filtros de admisión de aire del motor diferentes marcas uno el original y los otros tres de marcas alternas (Shogun, Samurai y Tecfil), adicional se utilizaron 4 marcas de bujías diferentes una de ellas la original del vehículo (champion) y las otras tres son de marcas alternas (Bosch, NGK y Denso), dentro de las pruebas de laboratorio se contempló realizar 3 calibraciones a los electrodo la primera a $0,7 \mathrm{~mm}$ la segunda a $0,85 \mathrm{~mm}$ y la tercera y última a $1 \mathrm{~mm}$. Las pruebas de laboratorio consisten en hacer todas las combinaciones posibles de filtros de aire, bujías y calibraciones para observar la tendencia del porcentaje de contaminación de gases de escape al medio ambiente, luego de obtener los resultados de las pruebas de laboratorio se tabularán los valores para determinar cuáles son los repuestos con los que el vehículo Chevrolet Sail 1.4L emite el menor porcentaje de gases de escape al medio ambiente. Estos datos son el resultado de nuestra investigación en el que consiste este proyecto y será nuestro aporte a la comunidad para que lo tomen como una opción al momento de realizar el mantenimiento de su vehículo y también será una opción al momento de hacer la revisión vehicular donde el ente regulador sensa la contaminación de gases emitida en el vehículo.

Palabras clave: repuestos; genérico; contaminación; admisión de aire; calibración; motor Abstract: This article aims to analyze if using generic spare parts in the vehicle Chevrolet Sail 1.4L specifically the engine air intake filter, the spark plugs and modifying the calibration of the spark plug electrodes we managed to reduce the percentage of emissions of pollutant gases to the environment. The importance of controlling emissions of polluting gases for the wellbeing of humans and our planet. At present the ozone layer is deteriorated products of harmful gases that cause the greenhouse effect. Combustion in the engines is the main cause of environmental 
pollution, so the electronics and other devices have been implemented to control and reduce the pollution generated by combustion in the engines. There were 192 laboratory tests where 4 different engine air intake filters were used, one with the original and the other with three other brands (Shogun, Samurai and Tecfil), 4 different brands of spark plugs were used, one of them being the original one Of the vehicle (champion) and the other three are of alternating marks (Bosch, NGK and Denso), within the laboratory tests it was contemplated to carry out 3 calibrations to the electrodes the first to $0.7 \mathrm{~mm}$ the second to $0.85 \mathrm{~mm}$ and the third And last to $1 \mathrm{~mm}$. Laboratory tests consist of making all possible combinations of air filters, spark plugs and calibrations to observe the trend of the percentage of exhaust gas contamination to the environment, after obtaining the results of the laboratory tests will tabulate the values for Determine which are the parts with which the Chevrolet Sail 1.4L vehicle emits the lowest percentage of exhaust gases to the environment. These data are the result of our research in which this project consists and will be our contribution to the community to take it as an option when performing the maintenance of your vehicle and will also be an option when doing the vehicle review where the regulator senses the contamination of gases emitted in the vehicle.

Key words: spare parts; generic; pollution; air intake; calibration; engine

\section{Introducción}

En este artículo se habla sobre la importancia de controlar las emisiones de los gases contaminantes por el bienestar de los seres humanos y de nuestro planeta. Actualmente la capa de ozono se encuentra deteriorada productos de gases nocivos que provocan el efecto invernadero. La combustión en los motores es la principal causa de contaminación ambiental por lo que se ha implementado la electrónica y demás dispositivos para controlar y reducir la contaminación generada por la combustión en los motores.

\section{Análisis Del Control De Emisiones Del Chevrolet Sail 1.4 L Año 2012}

El transporte automotor es una de las principales emisoras de gases contaminantes que son provocadas por la combustión de los motores, los daños causados son tanto al ser humano como también al ecosistema por el efecto invernadero.

Los gases dañinos al ser humano son los siguientes:

- Hidrocarburos no quemados (HC).

- Monóxido de carbono (CO).

- Óxidos de nitrógeno (NOx).

Por otro lado, los gases que afectan al ecosistema destruyendo la capa de ozono y provocando el efecto invernadero son los siguientes:

- Metano (CH4).

- Dióxido de carbono (CO2)

- Óxido nitroso (N2O). 
El desarrollo tecnológico de los países que tienen el mayor porcentaje de aportación a la emisión de gases contaminantes debido a la quema de los combustibles, el incremento de las emisiones de gases contaminantes a la atmosfera que repercute directamente en el deterioro de la calidad de vida que percibe la población es uno de principales problemas y reto a la vez que debe resolver por el bien de la sociedad. Sin duda la solución no está en eliminar el transporte ya que es una de las principales fuentes de la economía de los países, ya que se encarga de transportar a las personas además se encarga de movilizar los insumos y materias primas que son requeridas para la producción tanto de consumo masivo como del resto de la economía de los países a nivel mundial. Además, beneficia en la transformación de las relaciones de trabajo y grupos sociales al intercambiar e incorporar productos a aquellas localidades que se enlazan a las redes del transporte, y es influyente en la ubicación de centros urbanos y sus actividades.

El enfoque para tratar el tema de la emisión de los gases contaminantes debe efectuarse considerando tres variables de forma simultánea, la primera la calidad de combustible utilizado, tecnología en los vehículos y condiciones de uso de los vehículos, de tal forma que cualquier variación de alguno de estos tres factores va a influir de manera directa en la modificación del nivel de emisiones ${ }^{1}$.

Utilizando un combustible de mejor calidad con bajo contenido de azufre podemos reducir entre un 20 a 30\% las emisiones de gases contaminantes. En cuanto a la tecnología vehicular, se enfoca directamente en cambios de diseño de motor, condiciones de combustión utilizando la electrónica automotriz, los sistemas postratamiento y el control de perdidas evaporativas como por ejemplo la inclusión de un catalizador de oxidación, una recirculación de gases de escape e incluso una cámara de combustión de quemado rápido.

Y si a lo detallado anteriormente incluye una adecuada gestión del tránsito en las ciudades se podría reducir de cinco y diez veces las emisiones vehiculares.

\section{Antecedentes De La Contaminación Vehicular}

En la última década el fuerte crecimiento del parque automotor en el Ecuador y los pocos controles por parte de las autoridades competentes en el tema ambiental han generado un incremento significativo los niveles de contaminación. En la actualidad la mayor parte por no decir todos los fabricantes de automóviles ha construido diversos sistemas de control para reducir los niveles de contaminación ambiental.

El parque automotor es una de las principales fuentes emisoras de gases contaminantes que provienen de la combustión en los motores, esta contaminación proviene fundamentalmente del sistema de encendido y del sistema de alimentación, aunque también depende por otra parte del desgaste interno de sus componentes, calibración de balancines etc., y que afectan directamente el incremento de contaminación en el ambiente. La contaminación está acelerando la destrucción de la capa de ozono y por ende el efecto invernadero provoca el mayor y más rápido cambio climático en el Planeta.

\section{Justificación Del Análisis De Gases Del Vehículo Chevrolet Sail 1.4L}

\footnotetext{
${ }^{1}$ http://www.cubasolar.cu/biblioteca/Ecosolar/Ecosolar23/HTML/articulo02.htm
} 
La principal razón para el estudio del análisis del sistema de control de emisiones contaminantes del vehículo Chevrolet Sail 1.4L es determinar con que calidad de combustible, marca de filtro de aire, marca de bujías y calibración de los electrodos de las bujías tiene el menor porcentaje de contaminación de gases de escape al ambiente.

Después de efectuar dicho análisis se recomendará la utilización de los mejores elementos a utilizar a los propietarios de la marca de estos vehículos debido a que en la Ciudad de

Guayaquil se encuentra el nuevo Centro de Revisión Vehicular donde además de hacer la revisión del estado de los puntos claves del vehículo también se está analizando la cantidad de emisiones de gases contaminantes que expulsa el motor por lo que es necesario que los propietarios conozcan que afecta directamente al incremento y disminución de los gases contaminantes de un vehículo marca Chevrolet modelo Sail 1.4L 2012. Con esta investigación estaremos aportando a la sociedad es general disminuyendo la contaminación al medio ambiente.

\section{Objetivos}

\section{Objetivo General}

Analizar el sistema de control de emisiones del Chevrolet Sail 1,4L año 2012que se encuentra dentro de la Universidad Internacional del Ecuador Extensión Guayaquil a fin de determinar las emisiones contaminantes con su filtro de aire original, bujías originales y compararlas con filtros de aire y bujías alternas.

\section{Objetivos Específicos}

- Realizar un estudio general del sistema de Control de Emisiones del vehículo

- Chevrolet Sail 1,4L año 2012.

- Calcular con el equipo de diagnóstico de gases de escape los resultados obtenidos en las pruebas de laboratorio.

- Determinar con que calidad de repuestos emite menor cantidad de gases contaminantes a la atmosfera.

- Comprobar los resultados del análisis del sistema de control de emisiones del vehículo Chevrolet Sail 1,4L año 2012.

\section{Métodos}

\section{Funcionamiento Teórico Del Sistema}

Aquí se analiza los principales aspectos teóricos del funcionamiento del motor del vehículo Chevrolet Sail 1.4.L, también se estudia los tipos de gases contaminantes que expulsa producto de la combustión. El motor es de combustión interna de 4 tiempos con sistema de inyección electrónico multipunto. La característica del sistema de inyección electrónica del motor es el uso de inyectores electrónicos que son comandados por la unidad de control que sirve para suministrar el combustible necesario para el motor. El volumen de admisión de aire del motor, temperatura de refrigerante, temperatura de admisión de aire, relación de aceleración y 
desaceleración y otras condiciones son detectadas por sensores que envían señales al módulo de control para compararlos con los parámetros almacenados en la memoria y así se pueda calcular la cantidad de combustible que se va a suministrar a los inyectores, de esta manera se logra un ajuste de la relación aire/combustible para que cumpla con los requerimiento del motor. Otra de las características del motor es que dispone de analizadores de gases de escape que están permanentemente suministrando información sobre las proporciones de gases de contaminantes de los residuos de la combustión, de tal forma que se corrijan inmediatamente.

\section{Conceptos Básicos Del Motor}

\section{Relación Entre Aire Y Combustible}

La relación aire/combustible es el número que expresa la cantidad en masa o en volumen de aire aspirado por un motor de combustión para una cantidad unitaria de combustible. Para una combustión completa de la mezcla de aire y combustible las proporciones de la mezcla deben encontrarse dentro de la relación estequiométrico. En el caso de la gasolina se requieren 14,7 gramos de aire por cada gramo de combustible. La relación de aire y combustible $\chi$ (lambda) indica hasta qué punto la mezcla aire combustible efectivamente existente difiere de la cantidad de aire teóricamente necesaria.

\section{$\chi=$ masa de aire suministrada/masa de aire teóricamente necesaria}

Cuando la masa de aire admitida es igual a la masa de aire que debería admitirse tenemos como resultado la mezcla estequiométrico ideal. Dentro del funcionamiento estequiométrico la lambda tiene valor de 1 .

\section{Mezcla Rica}

Si la relación de aire y combustible es menor a la mezcla estequiométrico tenemos como resultado una mezcla rica, lo que significa que tenemos un exceso de combustible para la cantidad de aire existente. Esto nos da como resultado que una parte de combustible no podrá ser quemado en su totalidad y saldrán de los cilindros por el múltiple de escape y hacia al ambiente como $\mathrm{H}_{\mathrm{x}} \mathrm{C}_{\gamma} \mathrm{y}$ otra parte se podrán quemar parcialmente produciendo monóxido de carbono $(\mathrm{CO}) .^{2}$

\section{$\chi=$ masa de aire suministrada/masa de aire teóricamente necesaria}

Cuando la masa de aire admitida es menor a la masa de aire que debería admitirse tenemos como resultado la mezcla rica. Las consecuencias de una mezcla rica se la ven reflejada en un alto consumo de combustible, bajo rendimiento del motor y gran emanación de gases.

\section{Mezcla Pobre}

Cuando la relación de aire y combustible es mayor a la ideal entonces tendremos como resultado una mezcla pobre, lo que significa que tenemos una cantidad excesiva de aire lo que trae como consecuencia problemas de encendido.

\footnotetext{
${ }^{2}$ Manual del automóvil reparación y mantenimiento
} 


\section{$\chi=$ masa de aire suministrada/masa de aire teóricamente necesaria.}

Cuando la masa de aire admitida es mayor a la masa de aire que debería admitirse tenemos como resultado la mezcla pobre. Las consecuencias de una mezcla pobre son pérdidas de potencia, sobrecalentamiento, gran emisión de gases, autoencendido, mayor desgaste del motor $^{3}$.

\section{Origen De Los Gases De Combustión}

La energía química que contienen los combustibles luego de ser quemada en forma de calor produce trabajo, para que el combustible sea quemado es necesario que deba existir oxigeno (aire), esa necesidad de aire admitido varía de acuerdo con las necesidades del motor y el tipo de combustible. Cuando todo el combustible es quemado, todo el carbono (C) presente en el combustible reacciona con el oxígeno $(\mathrm{O} 2)$ formando dióxido de carbono $\mathrm{CO} 2$, adicional todo el hidrogeno $(\mathrm{H})$ que está presente en el combustible también reacciona con eloxígeno $(\mathrm{O})$ formando vapor de agua $\mathrm{H} \mathrm{O}$ y posteriormente todo el hidrogeno $\mathrm{H}$ existente 222en el aire admitido no participa en la reacción y este es expulsado de la misma manera.

Tabla 1. Gases producidos en la combustión.

\begin{tabular}{|c|c|}
\hline $\mathrm{H} \mathrm{O}_{2}$ & Vapor de agua \\
\hline $\mathrm{CO}_{2}$ & Dióxido de carbono \\
\hline $\mathrm{N} 2$ & Nitrógeno \\
\hline $\mathrm{CO}$ & Hidrocarburos \\
\hline $\mathrm{HxCy}$ & Oxido de nitrógeno, dióxido de nitrógeno etc. \\
\hline $\mathrm{NOx}$ & Hidrogeno \\
\hline $\mathrm{H} 2$ & Metano \\
\hline $\mathrm{CH} 4$ & Oxido de azufre, dióxido de azufre etc. \\
\hline $\mathrm{SOx}$ & Oxigeno \\
\hline $\mathrm{O} 2$ & \\
\hline
\end{tabular}

Autor: Marco Noroña

\section{Gases Contaminantes En El Vehículo}

El motor de combustión de un vehículo expulsa muchos gases al ambiente que son sustancias nocivas y otras que son sustancias inocuas.

\footnotetext{
${ }^{3}$ Tecnología del automóvil. Stuttgart.
} 


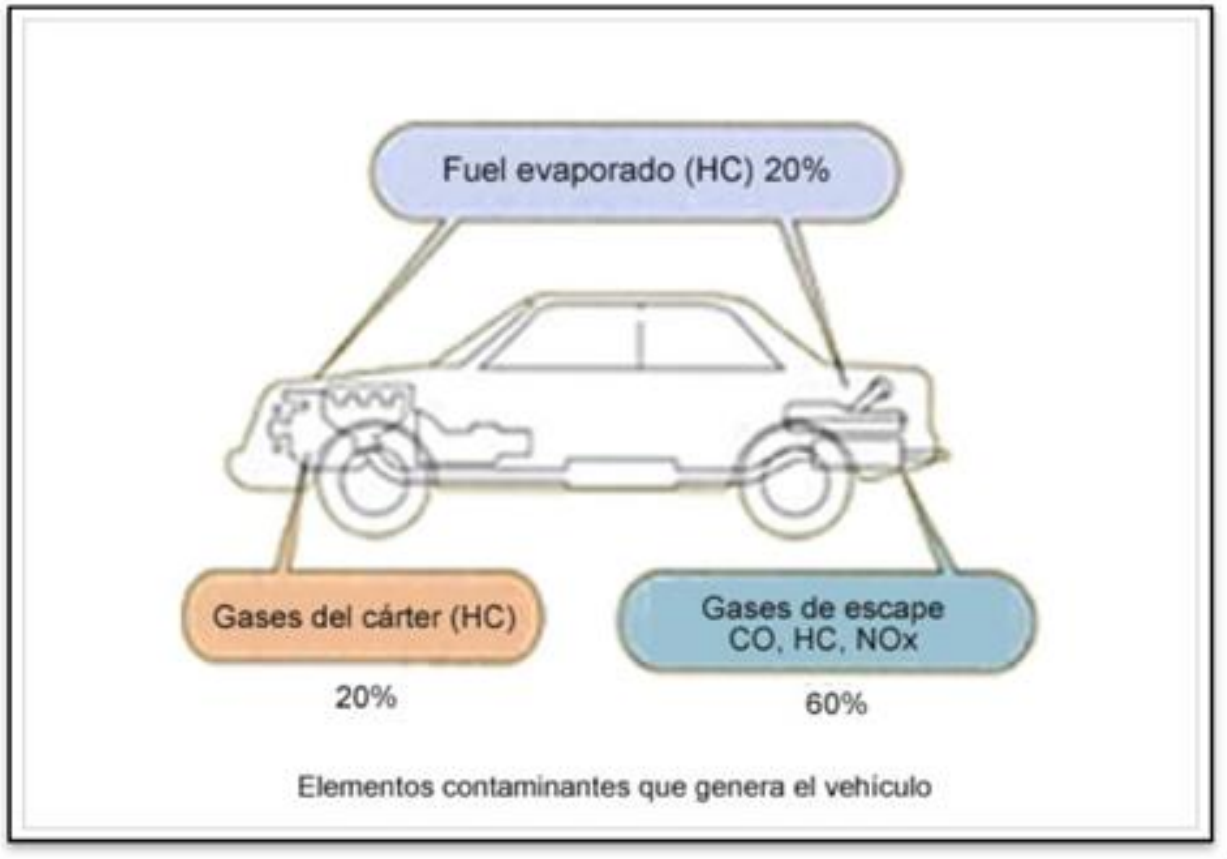

Figura 1. Gases contaminantes en el vehículo

Fuente: http://www.aficionadosalamecanica.net/emision-gases-escape.htm

De los principales gases que contaminan al ambiente son los siguientes:

Tabla 2. Principales gases que contaminan al ambiente

\begin{tabular}{|c|c|}
\hline Monóxido de carbono & $\mathrm{CO}$ \\
\hline Hidrocarburos & $\mathrm{HxCy}$ \\
\hline Óxido de nitrógeno, dióxido de nitrógeno etc. & $\mathrm{NOx}$ \\
\hline
\end{tabular}

Autor: Marco Noroña

\section{Monóxido De Carbono}

Esta sustancia nociva se produce cuando hay poco oxígeno en la cámara de combustión y en consecuencia no es posible la oxidación completa del carbono $(\mathrm{C})$ para formar el dióxido de carbono (CO) que no es un gas venenoso. El monóxido de carbono es el gas que se 2 considera en primer lugar en las regulaciones, básicamente este gas depende de la relación aire/combustible.

El monóxido es altamente toxico debido a la gran compatibilidad con la hemoglobina de la sangre reduciendo la oxigenación de los tejidos celulares, cuando el porcentaje de concentración de la carboxihemoglobina en la sangre supera el 50\% el cuerpo humano no consigue asimilar el oxígeno y por ende ocasiona la asfixia y posteriormente a muerte, este gas es un gas venenoso, inodoro e incoloro. 


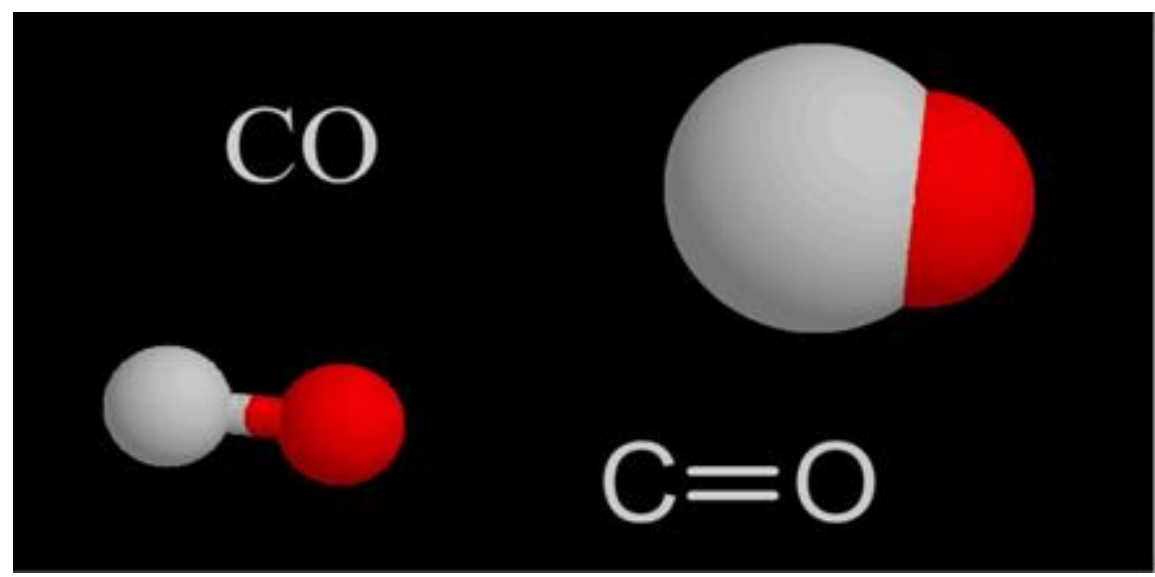

Figura 2. Monóxido de carbono

Fuente: http://listindiario.com/la-republica/2007/5/6/11908/Las-ciudades-se-ahogan-en- Monoxido-decarbono

El Monóxido de Carbono (CO) es un gas pobre en oxigeno por tal motivo cuanto mayor sea la cantidad de oxigeno contenida en la mezcla menor será el porcentaje de monóxido de carbono expulsado al ambiente. Otro factor determinante en la disminución del porcentaje de monóxido de carbono (CO) es la homogeneidad de la mezcla, mientas la mezcla aire/combustible sea lo más homogénea posible menor será el porcentaje de monóxido de carbono generado. Como habíamos hablado el monóxido de carbono es producto de una mala combustión, normalmente el valor correcto está comprendido entre el $0,5 \%$ y $2 \%$ siendo la unidad de medida el porcentaje en volumen. El monóxido de carbono en combinación con el oxígeno da como resultado la siguiente ecuación.

\section{$\mathrm{C}+\mathrm{O}$}

\section{Hidrocarburos}

Este gas también se produce por la falta de aire en la cámara de combustión ya que la falta de éste hace que la combustión sea incompleta y por ende se producen hidrocarburos sin quemar o quemados parcialmente, y también se los conoce como smog en la ciudad. Por tanto, la alta cantidad de hidrocarburos no quemados pueden ser nocivos para la salud humana provocando un olor desagradable e irritación en los ojos y también se lo relaciona con la lluvia ácida.

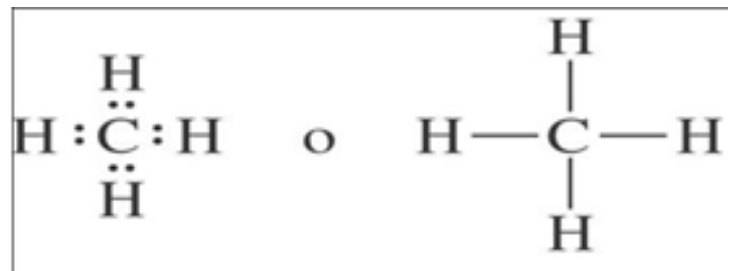

metano

Figura 3. Metano

Fuente: http://www.mecanicavirtual.com.ar/p/todo-sobre-motores.html 
El principal gas de estas características de polución en la atmosfera es el metano $(\mathrm{CH} 4)$ que representa el $85 \%$, el otro $15 \%$ están concentrados en mayor cantidad en etano, n-butano, etileno, benceno, propano, acetileno, isopentano y $\mathrm{n}$-pentano y en menor cantidad en cis-2buteno, cis-2-penteno, trans-2-buteno y metilacetileno. El valor de la concentración de HxCy (x, $\mathrm{y}$, son variables), en los gases de escape es altamente elevado cuando el motor está funcionando en desaceleración es decir con alta depresión en el múltiple de admisión.

La unidad de medida de los hidrocarburos no quemados es la ppm (partes por millón de partes). La conversión es: $1 \%=10000 \mathrm{ppm}$. Es utilizada la ppm debido a que la concentración de los hidrocarburos no quemados (HC) en los gases de escape es muy pequeña. Una indicación alta del hidrocarburo no quemado (HC) indica lo siguiente:

1. Mezcla rica, el óxido de carbono (CO) también da un valor alto.

2. Mala combustión de mezcla pobre.

3. Escape o aceite contaminado.

El valor normal está comprendido entre 100 y 400 ppm.

\section{$\mathrm{HC}+\mathrm{O} \square \mathrm{H2} \mathbf{O}$ (vapor de agua)}

\section{Óxidos De Nitrógeno}

El nitrógeno, que es el principal componente del aire, no se combina con el aire a las temperaturas normales, pero cuando tiene aportaciones de energía muy fuertes que generalmente ocurren en los procesos de combustión debido a las altas presiones y temperaturas en el interior del motor se produce una reacción química en la que se forma monóxido de nitrógeno. Al salir de los cilindros vuelve a combinarse con el oxígeno para formar dióxido de nitrógeno. El monóxido de nitrógeno y el dióxido de nitrógeno son gases estables y se les designa con las siglas NOx.

Estos gases en altas concentraciones originan irritaciones al aparato respiratorio, síntomas 4 de envenenamiento y si se respira demasiado tiempo puede causar hasta la muerte ${ }^{4}$. El óxido de nitrógeno es un gas incoloro y sin olor, en el aire se transforma lentamente en dióxido de nitrógeno (NO2), El NO2 es en su forma pura un gas venenoso pardo rojizo de olor penetrante. Adicional este gas también es el causante de daños forestales y junto con los hidrocarburos de la formación del smog.

\section{EI Sistema De Escape}

El sistema de escape tiene la función principal de conducir los gases quemados producto de la combustión desde el cabezote o culata hasta el exterior del vehículo. Adicional a esto el sistema de escape también tiene la función de reducir el ruido que es producido por los gases de escape debido a las ondas que es producido por dichos gases. El sistema de escape tiene influencia directamente sobre el funcionamiento del motor, cuando se utiliza el escape libre la potencia del motor aumenta debido a que los gases quemados producidos por la combustión son

\footnotetext{
4 Técnica de gases de escape para motores de gasolina
} 
evacuados más rápidos desde la cámara de combustión, pero si el sistema de escape se encuentra obstruido el motor tiende a perder potencia.

$\checkmark$ Colector de escape.

$\checkmark$ Conductos de evacuación.

$\checkmark$ Convertidor catalítico.

$\checkmark$ Silenciador.

\section{Sistema De Control De Emisiones Contaminantes En El Automóvil}

Los motores de combustión interna tienen varias formas de controlar y reducir la emisión de los gases contaminantes al ambiente, a continuación, hablaremos de los siguientes sistemas de control:

1. Sistema de ventilación positiva del cárter.

2. Sistema de control de emisiones evaporativas.

3. Sistema de recirculación parcial de los gases de escape

4. Convertidor catalítico.

\section{Pruebas De Laboratorio De Las Emisiones De Los Gases Contaminantes Del Vehículo Chevrolet Sail 1.4l}

Antes de comenzar a realizar las pruebas de laboratorio de las emisiones de gases contaminantes se procedió a realizar dos pruebas en el motor del vehículo las cuales consisten en la medición de la presión de compresión de los cilindros y la medición del vacío del motor.

\section{Medición De La Presión De Compresión}

Es el valor de la presión en el punto muerto superior medido en el interior de la cámara de combustión mediante un manómetro que se lo coloca en el orifico donde va instalada la bujía. El valor medido nos indica el estado de degaste y eficacia del cilindro.

Dicha prueba se la efectúa desmontando las bujías y colocando un manómetro mediante una manguera que se acopla en la rosca de alojamiento de la bujía, luego se da arranque al motor con el pedal del acelerador accionado a su máxima posición y obtendremos los resultados en el manómetro, esta prueba se la debe realizar con el motor a temperatura de operación.

En este motor obtuvimos los siguientes resultados de la presión de compresión:

Tabla 3. Valores de compresión de cilindros

\begin{tabular}{cc}
\hline \# de cilindro & $\begin{array}{c}\text { Valor de presión de } \\
\text { comprensión }\end{array}$ \\
\hline Cilindro \# 1 & $185 \mathrm{psi}$ \\
Cilindro \# 2 & $190 \mathrm{psi}$ \\
Cilindro \# 3 & $180 \mathrm{psi}$ \\
Cilindro \# 4 & $180 \mathrm{psi}$ \\
\hline
\end{tabular}

Autor: Marco Noroña 


\section{Medición De Vacío Del Motor}

Se procedió a realizar la prueba de vacío en el motor del vehículo Chevrolet Sail antes de comenzar a realizar las pruebas de laboratorio para saber el estado interno de desgaste, la condición de tener vacío dentro del múltiple de admisión nos permita saber si el motor está en óptimas condiciones de trabajo. Para realizar la prueba de vacío fue necesario utilizar un vacuometro que es la herramienta que mide vacío en unidades de pulgadas de mercurio. El valor del vacío del motor es de 18 pulgadas de mercurio, con este valor determinamos que el motor se encuentra en condiciones normales de funcionamiento.

Para realizar las pruebas de laboratorio se utilizaron los siguientes equipos herramientas y repuestos.

\section{Analizador De Gas Modelo Brainbee Gs-688}

Este equipo es uno de los equipos más completos y versátiles del mercado puesto que incluye pantallas lcd para las lecturas de los gases medidos, así como los datos de las RPM y temperatura de aceite del motor, también cuenta con una impresora térmica para la impresión de reportes con los valores Características principales:

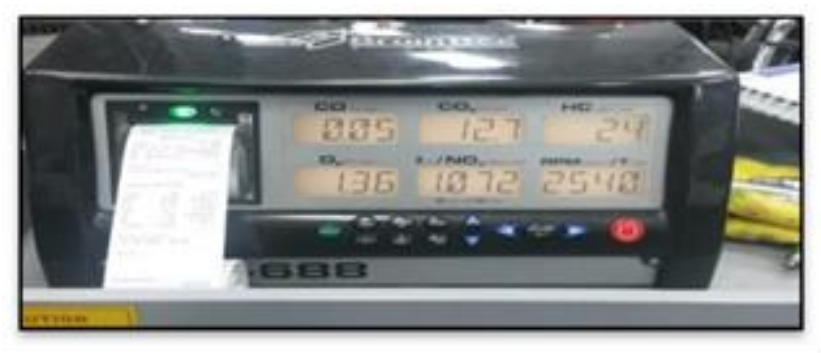

Figura 4. Analizador de gas BRAIN BEE AGS-688 Fuente: Marco Noroña

El analizador de gas BrainBee AGS-688 cuenta con un accesorio adicional modelo MGT300 que sirve para conectarse inalámbricamente por medio de su receptor de radio. El accesorio MGT-300 provee al analizador de gases las lecturas de RPM y temperatura del motor de cualquier vehículo sea de gasolina o diésel, de 2 o 4 tiempos y de hasta 12 cilindros, este accesorio trabaja conectándolo a la batería del motor.

\section{Herramientas De Taller}

Para realizar las pruebas de laboratorio se utilizó varias herramientas básicas de taller, continuación, las detallamos.

Tabla 4. Herramientas utilizadas

\begin{tabular}{llll}
\hline Ítem & Descripción & Cant & Uso \\
\hline $\mathbf{1}$ & Llave de bujías & 1 & $\begin{array}{c}\text { Se la utilizó para la } \\
\text { extracción de las bujías }\end{array}$ \\
\hline
\end{tabular}




\begin{tabular}{|c|c|c|c|}
\hline 2 & Llave allen $3 / 16^{\prime \prime}$ & 1 & $\begin{array}{c}\text { Se la utilizó para } \\
\text { desmontar la tapa de válvulas }\end{array}$ \\
\hline 3 & Calibrador de galgas & 1 & $\begin{array}{l}\text { Se la utilizó para calibrar } \\
\text { los electrodos de las bujías }\end{array}$ \\
\hline 4 & $\begin{array}{l}\text { Palanca de media } \\
\text { fuerza }\end{array}$ & 1 & $\begin{array}{c}\text { Se la utilizó para la } \\
\text { extracción de las bobinas }\end{array}$ \\
\hline 5 & $\begin{array}{l}\text { Extensión de palanca } \\
\text { de media fuerza }\end{array}$ & 1 & $\begin{array}{c}\text { Se la utilizó para la } \\
\text { extracción de las bobinas }\end{array}$ \\
\hline 6 & Dado de copa \#10 & 1 & $\begin{array}{c}\text { Se la utilizó para la } \\
\text { extracción de las bobinas }\end{array}$ \\
\hline 7 & $\begin{array}{l}\text { Reservorio de } \\
\text { combustible }\end{array}$ & 1 & $\begin{array}{l}\text { Se lo utilizó para comprar } \\
\text { combustible }\end{array}$ \\
\hline
\end{tabular}

Autor: Marco Noroña

\section{Repuestos Utilizados}

Para realizar las pruebas de laboratorio se utilizaron los repuestos que se detalla a continuación:

\section{Bujías}

La bujía es un elemento que produce el encendido de la mezcla aire/combustible en la cámara de combustión, mediante la chispa. La bujía tiene 2 funciones primarias, la primera es de inflamar la mezcla aire/combustible y la segunda de disipar el calor generado en la cámara de combustión hacia el sistema de refrigeración del motor. Para efectos de las pruebas se utilizaron 4 marcas de bujías que más se comercializan en el mercado para el vehículo Chevrolet Sail 1.4LCU.
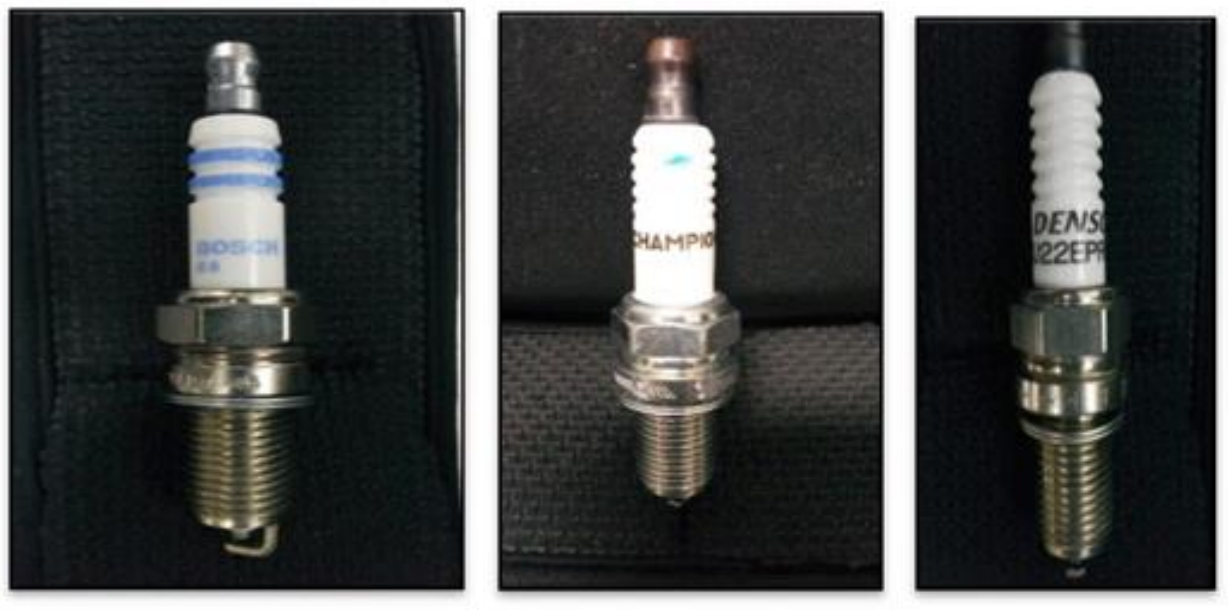


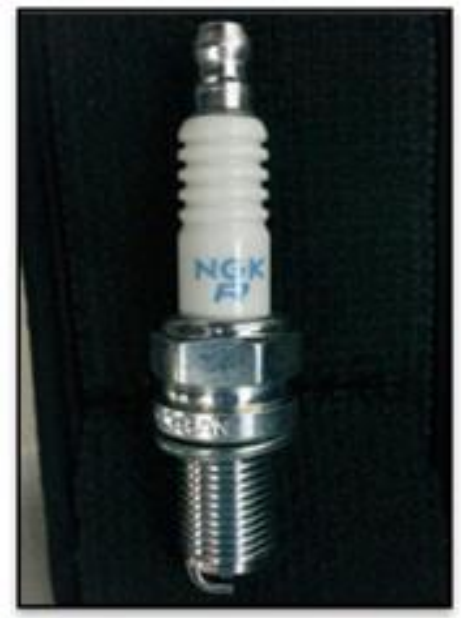

Figura 5. Bujías Marca Bosch, Denso, Ngk Y Champion Autor: Marco Noroña

\section{Filtros De Aire De Motor}

El filtro de aire es un elemento que retiene partículas sólidas como el polvo, y evitan que estas ingresen al interior del motor por medio del múltiple de admisión y puedan provocar desgaste prematuro en el interior del cilindro. Este filtro de aire está fabricado a base de papel plegado filtrante, el tamaño del papel filtrante determina la capacidad de filtrado que puede realizar y por ende podemos determinar la calidad del mismo.

Para efectos de las pruebas de laboratorio se utilizaron 4 diferentes marcas de filtros de aire tomando como parámetro una pequeña encuesta (ver anexo) realizada a 20 personas de cuáles son los filtros de aire de motor que más utilizan en los vehículos Chevrolet Sail 1.4L.

De los resultados de la encuesta se determinó utilizar los filtros de las siguientes marcas:

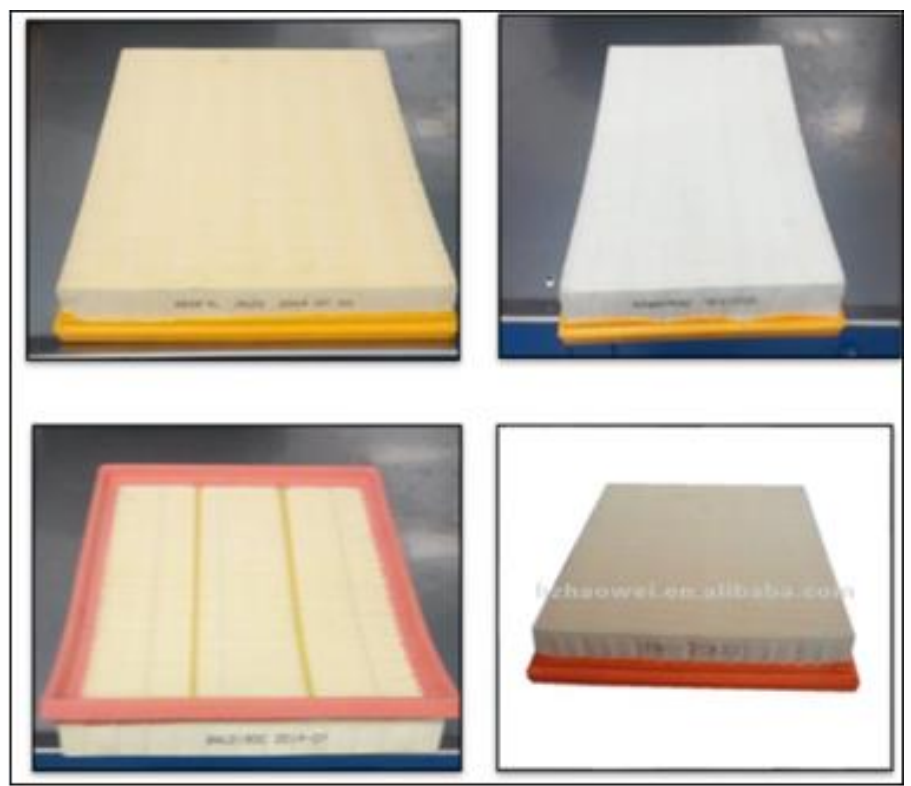

Figura 6. Filtros De Aire Marca Redfil, Samurai, Shogun Y Shangai Autor: Marco Noroña 


\section{Análisis De Resultados}

\section{Medición De Resultados Utilizando Combustible De 92 Octanos A 700rpm}

A continuación, detallamos la tabla de valores con los resultados obtenidos luego de hacer las pruebas de laboratorio utilizando el filtro de aire original con las cuatro diferentes marcas de bujías a tres calibraciones de electrodos diferentes a 700rpm utilizando combustible de 9 octanos.

En esta tabla (tabla 6.1) vamos a obtener como resultado con que bujía y a que calibración del electrodo emite menor emisión de gases el vehículo Chevrolet Sail 1.4 a 700rpm.

Tabla 5. Análisis de resultado de valores de emisiones de gases utilizando filtro de aire original vs variables

\begin{tabular}{|c|c|c|c|c|c|c|c|c|c|c|c|c|c|}
\hline Combustible & \multicolumn{13}{|c|}{ Gasolina súper de 92 octanos } \\
\hline Filtro de aire & \multicolumn{13}{|c|}{ ORIGINAL } \\
\hline Bujias & \multicolumn{3}{|c|}{ ORIGINAL } & \multicolumn{3}{|c|}{$\mathrm{BOSCH}$} & \multicolumn{3}{|c|}{ NGK } & \multicolumn{3}{|c|}{ DENSO } & \\
\hline RPM & \multicolumn{13}{|c|}{ RALENTÍ } \\
\hline $\begin{array}{l}\text { Calibración } \\
\text { Electrodo }\end{array}$ & $0,7 \mathrm{~mm}$ & $\begin{array}{c}0,85 \mathrm{~m} \\
\mathrm{~m}\end{array}$ & $1 \mathrm{~mm}$ & $0,7 \mathrm{~mm}$ & $\begin{array}{c}0,85 \mathrm{~m} \\
\mathrm{~m}\end{array}$ & $1 \mathrm{~mm}$ & $0,7 \mathrm{~mm}$ & $\begin{array}{c}0,85 \mathrm{~m} \\
\mathrm{~m}\end{array}$ & $1 \mathrm{~mm}$ & $0,7 \mathrm{~mm}$ & $\begin{array}{c}0,85 \mathrm{~m} \\
\mathrm{~m}\end{array}$ & $1 \mathrm{~mm}$ & $\begin{array}{l}\text { Unidad de } \\
\text { medida }\end{array}$ \\
\hline co & 0,01 & 0 & 0,02 & 0,02 & 0,02 & 0,01 & 0 & 0 & 0,02 & 0,02 & 0,01 & 0,02 & (\%Vol) \\
\hline $\mathrm{CO} 2$ & 14,5 & 14,2 & 14,2 & 13,7 & 15,3 & 15 & 14,5 & 15,3 & 13,9 & 15,5 & 15,2 & 15,1 & (\%Vol) \\
\hline HC & 28 & 3 & 20 & 16 & 30 & 1 & 8 & 10 & 21 & 0 & 36 & 18 & (ppmVol) \\
\hline 02 & 0,11 & 0,06 & 0,06 & 1,52 & 0,08 & 0,12 & 0,08 & 0,08 & 0,05 & 0,08 & 0,11 & 0,08 & (\%Vol) \\
\hline Lambda & 1,004 & 1,003 & 1,002 & 1,076 & 1,002 & 1,005 & 1,004 & 1,003 & 1,001 & 1,003 & 1,003 & 1,002 & $(-)$ \\
\hline
\end{tabular}

Autor: Marco Noroña

Luego de las pruebas de laboratorios determinamos mediante los valores arrojados que la bujía que emite menor emisión de gases es la BOSCH calibrado sus electrodos a $1 \mathrm{~mm}$ a $70 \mathrm{rpm}$ utilizando el filtro de aire original. A continuación, se detallan las tabulaciones de los datos mediante las siguientes gráficas con las diferentes calibraciones de los electrodos de las bujías. 


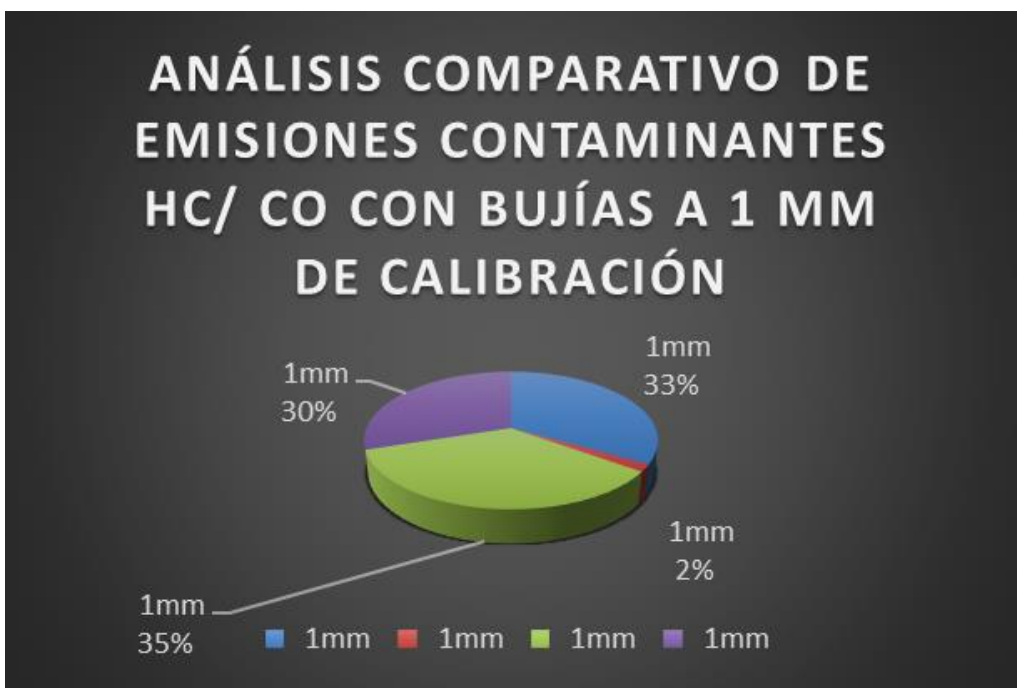

Figura 7. Análisis de Gases a 700 rpm combustible 92 octanos variable bujía calibración electrodo a 1 mm con filtro de aire original

Con la información de la primera tabla (tabla 5) donde nos arroja como resultado que la bujía marca BOSCH es la que emite menor contaminación al ambiente calibrado sus electrodos a $1 \mathrm{~mm}$ y a 700rpm, ahora en la siguiente tabla (tabla 6) se va a combinar la bujía marca BOSCH calibrados sus electrodos a $1 \mathrm{~mm}$ con cuatro marcas de filtros de aire diferentes, uno es el original del vehículo y los otros tres son alternos.

Tabla 6. Análisis de resultado de valores de emisiones de gases utilizando la bujía marca BOSCH vs varias variables de filtro

\begin{tabular}{|c|c|c|c|c|c|c|c|c|c|c|c|c|c|}
\hline Combustible & \multicolumn{13}{|c|}{ Gasolina súper de 92 octanos } \\
\hline BUJIA & \multicolumn{13}{|c|}{$\mathrm{BOSCH}$} \\
\hline Filtro de aire & \multicolumn{3}{|c|}{ (ORIGINAL) } & \multicolumn{3}{|c|}{ SAMURAI } & \multicolumn{3}{|c|}{ SHOGUN } & \multicolumn{3}{|c|}{ REDFIL } & \\
\hline RPM & \multicolumn{13}{|c|}{700} \\
\hline $\begin{array}{l}\text { Calibración } \\
\text { Electrodo }\end{array}$ & $0,7 \mathrm{~mm}$ & $\begin{array}{c}0,85 \mathrm{~m} \\
\mathrm{~m}\end{array}$ & $1 \mathrm{~mm}$ & $0,7 \mathrm{~mm}$ & $\begin{array}{c}0,85 \mathrm{~m} \\
\mathrm{~m}\end{array}$ & $1 \mathrm{~mm}$ & $0,7 \mathrm{~mm}$ & $\begin{array}{c}0,85 \mathrm{~m} \\
\mathrm{~m}\end{array}$ & $1 \mathrm{~mm}$ & $0,7 \mathrm{~mm}$ & $\begin{array}{c}0,85 \mathrm{~m} \\
\mathrm{~m}\end{array}$ & $1 \mathrm{~mm}$ & $\begin{array}{l}\text { Unidad de } \\
\text { medida }\end{array}$ \\
\hline co & 0,02 & 0,01 & 0,02 & 0 & 0 & 0 & 0,02 & 0 & 0,01 & 0,01 & 0,01 & 0,02 & $(\% \mathrm{Vol})$ \\
\hline $\mathrm{CO} 2$ & 15,5 & 15,2 & 15,1 & 12,6 & 15,3 & 15,2 & 15,3 & 15,3 & 15 & 13 & 15,2 & 15 & $(\% \mathrm{Vol})$ \\
\hline HC & 0 & 36 & 18 & 0 & 8 & 8 & 1 & 6 & 20 & 15 & 8 & 28 & $(\mathrm{ppmVol})$ \\
\hline 02 & 0,08 & 0,11 & 0,08 & 2,41 & 0,06 & 0,07 & 0,36 & 0,05 & 0,09 & 1,89 & 0,07 & 0,07 & $(\% \mathrm{Vol})$ \\
\hline Lambda & 1,003 & 1,003 & 1,002 & 1,134 & 1,002 & 1,003 & 1,016 & 1,002 & 1,003 & 1,101 & 1,003 & 1,002 & $(-)$ \\
\hline
\end{tabular}

Autor: Marco Noroña

Luego de las pruebas de laboratorios determinamos mediante los valores arrojados que el filtro de aire marca SAMURAI es el que emite menor emisión de gases en combinación con la 
bujía marca BOSCH calibrado sus electrodos a $1 \mathrm{~mm}$ a $700 \mathrm{rpm}$. A continuación, se detallan las tabulaciones de los datos mediante las siguientes gráficas con los diferentes filtros de aire

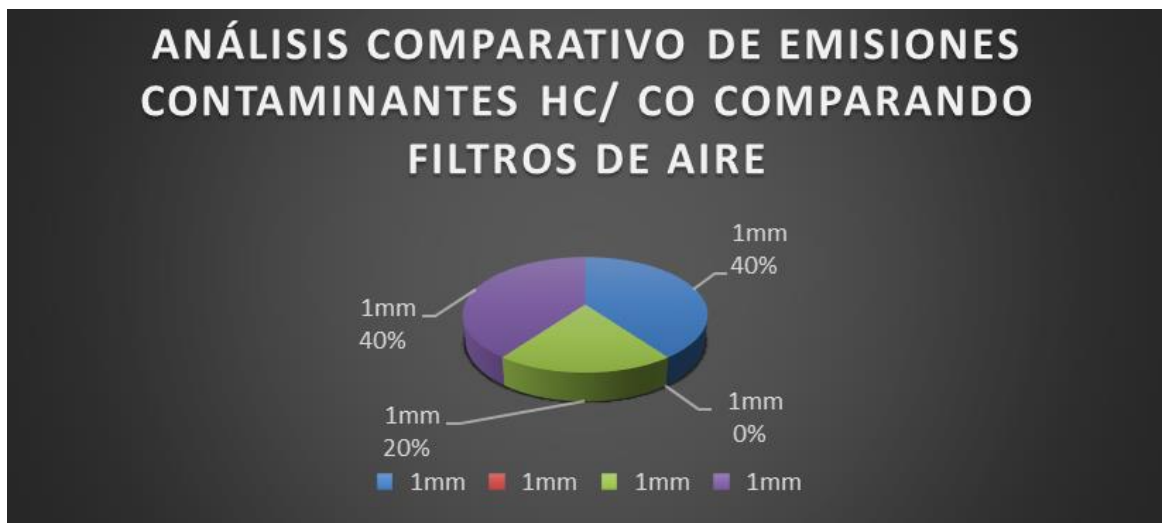

Figura 8. Análisis de Gases a 700rpm combustible 92 octanos variable filtros de aire con bujía BOSCH electrodo a $1 \mathrm{~mm}$

Autor: Marco Noroña

\section{Medición De Resultados Utilizando Combustible De 92 Octanos A 2500rpm}

A continuación, detallamos la tabla de valores con los resultados obtenidos luego de hacer las pruebas de laboratorio utilizando el filtro de aire original con las cuatro diferentes marcas de bujías a tres calibraciones de electrodos diferentes a $2500 \mathrm{rpm}$ utilizando combustible de 92 octanos. En esta tabla (tabla 7) vamos a obtener como resultado con que bujía y a que calibración del electrodo emite menor emisión de gases el vehículo Chevrolet Sail 1.4 a $2500 \mathrm{rpm}$.

Tabla 7. Análisis de resultado de valores de emisiones de gases utilizando filtro de aire original vs varias variables de bujías - Autor. Marco Noroña

\begin{tabular}{|c|c|c|c|c|c|c|c|c|c|c|c|c|c|}
\hline Combustible & \multicolumn{13}{|c|}{ Gasolina súper de 92 octanos } \\
\hline Filtro de aire & \multicolumn{13}{|c|}{ ORIGINAL } \\
\hline Bujias & \multicolumn{3}{|c|}{ ORIGINAL (CHAMPION) } & \multicolumn{3}{|c|}{$\mathrm{BOSCH}$} & \multicolumn{3}{|c|}{ NGK } & \multicolumn{3}{|c|}{ DENSO } & \\
\hline RPM & \multicolumn{13}{|c|}{2500} \\
\hline Descripción & $0,7 \mathrm{~mm}$ & $\begin{array}{c}0,85 \mathrm{~m} \\
\mathrm{~m}\end{array}$ & $1 \mathrm{~mm}$ & $0,7 \mathrm{~mm}$ & $\begin{array}{c}0,85 \mathrm{~m} \\
\mathrm{~m}\end{array}$ & $1 \mathrm{~mm}$ & $0,7 \mathrm{~mm}$ & $\begin{array}{c}0,85 \mathrm{~m} \\
\mathrm{~m}\end{array}$ & $1 \mathrm{~mm}$ & $0,7 \mathrm{~mm}$ & $\begin{array}{c}0,85 \mathrm{~m} \\
\mathrm{~m}\end{array}$ & $1 \mathrm{~mm}$ & $\begin{array}{l}\text { Unidad de } \\
\text { medida }\end{array}$ \\
\hline co & 0,01 & 0,04 & 0,05 & 0 & 2,02 & 0,01 & 0,01 & 0,04 & 0,1 & 0 & 0,01 & 0,06 & (\%Vol) \\
\hline $\mathrm{CO} 2$ & 13,9 & 14,1 & 12,7 & 14,9 & 13,2 & 15 & 14,5 & 14,5 & 13,9 & 13,1 & 15,2 & 15 & ( \% Vol) \\
\hline HC & 6 & 44 & 24 & 6 & 221 & 1 & 5 & 13 & 23 & 7 & 15 & 14 & (ppmVol) \\
\hline $\mathrm{O2}$ & 0,96 & 0,05 & 1,36 & 0,07 & 0,14 & 0,08 & 0,08 & 0,08 & 0,06 & 1,44 & 0,21 & 0,18 & (\%Vol) \\
\hline Lambda & 1,048 & 0,999 & 1,072 & 1,003 & 0,939 & 1,003 & 1,003 & 1,002 & 0,999 & 1,077 & 1,009 & 1,006 & $(-)$ \\
\hline
\end{tabular}


Luego de las pruebas de laboratorios determinamos mediante los valores arrojados que la bujía que emite menor emisión de gases es la BOSCH calibrado sus electrodos a 1mm a 2500 rpm utilizando el filtro de aire original. A continuación, se detallan las tabulaciones de los datos mediante las siguientes gráficas con las diferentes calibraciones de los electrodos de las bujías.

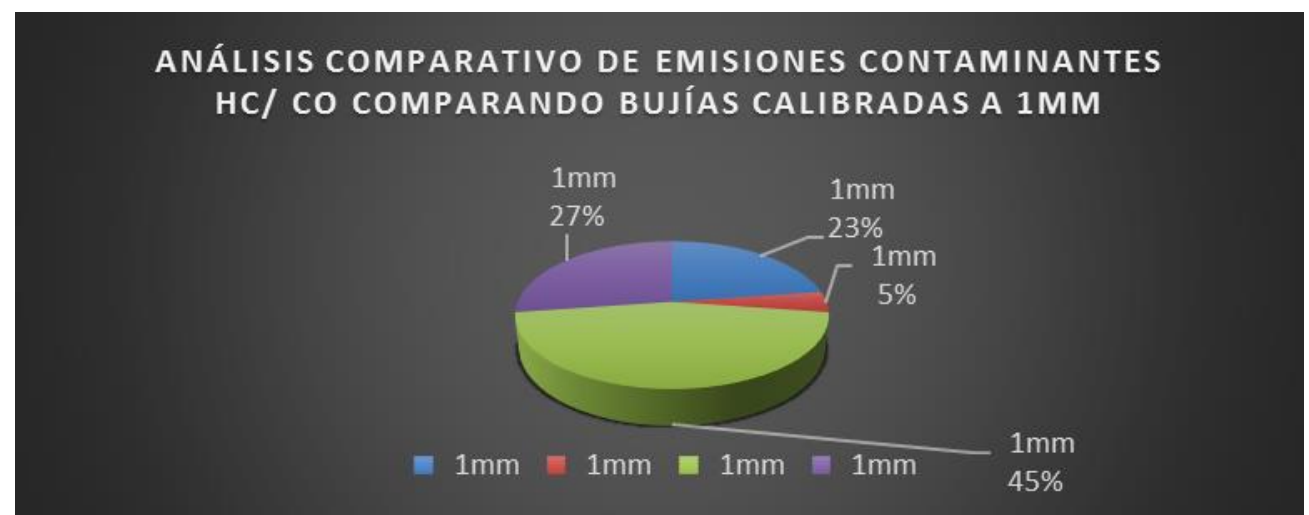

Figura 9. Análisis de Gases a 2500rpm combustible 92 octanos variable bujía calibración electrodo a 1mm con filtro de aire original

Autor: Marco Noroña

Con la información de la primera tabla (tabla 7) donde nos arroja como resultado que la bujía marca BOSCH es la que emite menor contaminación al ambiente calibrado sus electrodos a $1 \mathrm{~mm}$ y a $2500 \mathrm{rpm}$ ahora en la siguiente tabla (tabla 8) vamos a combinar la bujía marca BOSCH calibrados sus electrodos a $1 \mathrm{~mm}$ con cuatro marcas de filtros de aire diferentes, uno es el original del vehículo y los otros tres son alternos.

Tabla 8. Análisis de resultado de valores de emisiones de gases utilizando la bujía marca BOSCH vs varias variables

\begin{tabular}{|c|c|c|c|c|c|c|c|c|c|c|c|c|c|}
\hline Combustible & \multicolumn{13}{|c|}{ Gasolina súper de 92 octanos } \\
\hline BUJIA & \multicolumn{13}{|c|}{$\mathrm{BOSCH}$} \\
\hline Filtro de aire & \multicolumn{3}{|c|}{ ORIGINAL } & \multicolumn{3}{|c|}{ SAMURAI } & \multicolumn{3}{|c|}{ SHOGUN } & \multicolumn{3}{|c|}{ REDFIL } & \\
\hline RPM & \multicolumn{13}{|c|}{2500} \\
\hline Descripción & $0,7 \mathrm{~mm}$ & $\begin{array}{c}0,85 \mathrm{~m} \\
\mathrm{~m}\end{array}$ & $1 \mathrm{~mm}$ & $0,7 \mathrm{~mm}$ & $\begin{array}{c}0,85 \mathrm{~m} \\
\mathrm{~m}\end{array}$ & $1 \mathrm{~mm}$ & $0,7 \mathrm{~mm}$ & $\begin{array}{c}0,85 \mathrm{~m} \\
\mathrm{~m}\end{array}$ & $1 \mathrm{~mm}$ & $0,7 \mathrm{~mm}$ & $\begin{array}{c}0,85 \mathrm{~m} \\
\mathrm{~m}\end{array}$ & $1 \mathrm{~mm}$ & $\begin{array}{l}\text { Unidad de } \\
\text { medida }\end{array}$ \\
\hline co & 0 & 2,02 & 0,01 & 0,15 & 0,03 & 0,06 & 0,01 & 0,01 & 0,02 & 0,03 & 0,03 & 0,05 & ( \% Vol ) \\
\hline $\mathrm{CO} 2$ & 14,9 & 13,2 & 15 & 15,1 & 14,9 & 14,6 & 15,2 & 15 & 14,2 & 14,7 & 13,7 & 13,7 & ( \% Vol) \\
\hline HC & 6 & 221 & 1 & 34 & 4 & 8 & 1 & 2 & 18 & 45 & 71 & 34 & (ppmVol) \\
\hline O2 & 0,07 & 0,14 & 0,08 & 0,11 & 0,08 & 0,07 & 0,14 & 0,08 & 0,53 & 0,09 & 0,23 & 0,36 & (\%Vol) \\
\hline Lambda & 1,003 & 0,939 & 1,003 & 0,999 & 1,003 & 1,001 & 1,006 & 1,003 & 1,025 & 1,002 & 1,008 & 1,015 & $(-)$ \\
\hline
\end{tabular}

Autor: Marco Noroña

Luego de las pruebas de laboratorios determinamos mediante los valores arrojados que el filtro de aire ORIGINAL es el que emite menor emisión de gases en combinación con la bujía 
marca BOSCH calibrado sus electrodos a 1mm a $2500 \mathrm{rpm}$. A continuación, se detallan las tabulaciones de los datos mediante las siguientes gráficas con los diferentes filtros de aire.

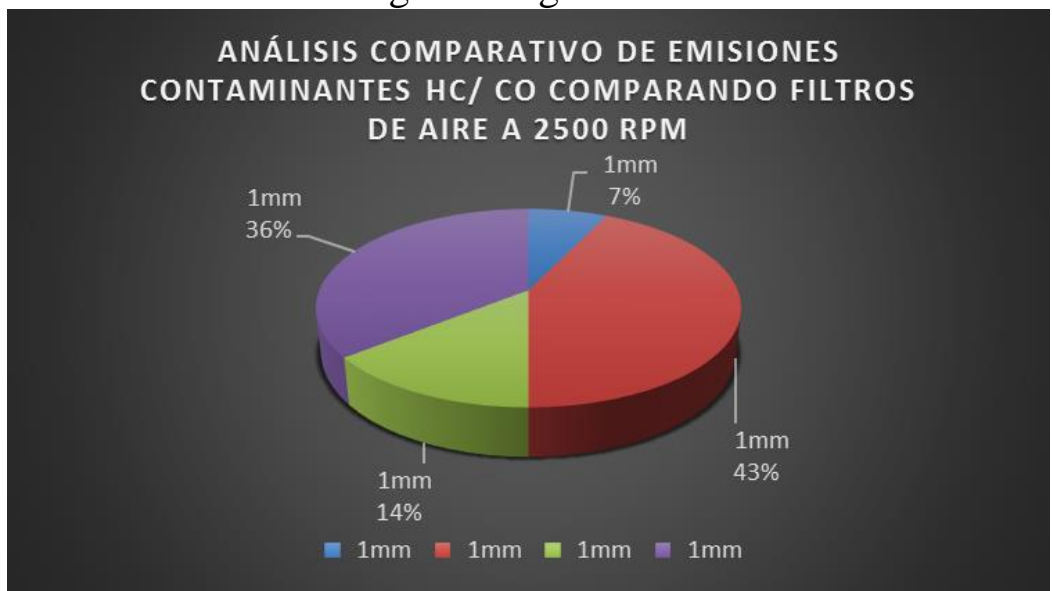

Figura 10. Análisis de Gases a 2500rpm combustible 92 octanos variable filtros de aire con bujía BOSCH electrodo a 1 mm - Autor: Marco Noroña

\section{Medición De Resultados Utilizando Combustible De 87 Octanos A 700rpm}

A continuación, detallamos la tabla de valores con los resultados obtenidos luego de hacer las pruebas de laboratorio utilizando el filtro de aire original con las cuatro diferentes marcas de bujías a tres calibraciones de electrodos diferentes a 700rpm utilizando combustible de 87 octanos. En esta tabla (tabla 6.5) vamos a obtener como resultado con que bujía y a que calibración del electrodo emite menor emisión de gases el vehículo Chevrolet Sail 1.4 a 700rpm.

Tabla 9. Análisis de resultado de valores de emisiones de gases utilizando la bujía marca BOSCH vs varias variables

\begin{tabular}{|c|c|c|c|c|c|c|c|c|c|c|c|c|c|}
\hline Combustibl & \multicolumn{13}{|c|}{ Gasolina súper de 87 octanos } \\
\hline $\begin{array}{c}\text { Filtro de } \\
\text { aire }\end{array}$ & \multicolumn{13}{|c|}{ ORIGINAL } \\
\hline Bujias & \multicolumn{3}{|c|}{ ORIGINAL (CHAMPION) } & \multicolumn{3}{|c|}{$\mathrm{BOSCH}$} & \multicolumn{3}{|c|}{ NGK } & \multicolumn{3}{|c|}{ DENSO } & \\
\hline RPM & \multicolumn{13}{|c|}{700} \\
\hline Descripción & $0,7 \mathrm{~mm}$ & $0,85 \mathrm{~mm}$ & $1 \mathrm{~mm}$ & $0,7 \mathrm{~mm}$ & $0,85 \mathrm{~mm}$ & $\mathrm{~mm}$ & $0,7 \mathrm{~mm}$ & $0,85 \mathrm{~mm}$ & $1 \mathrm{~mm}$ & $0,7 \mathrm{~mm}$ & $0,85 \mathrm{~mm}$ & $\mathrm{~mm}$ & $\begin{array}{l}\text { Unidad de } \\
\text { medida }\end{array}$ \\
\hline $\mathrm{CO}$ & 0,01 & 0 & 0,01 & 0 & 0 & 0 & 0,02 & 0 & 0 & 0,01 & 0 & 0,01 & (\% $\% \mathrm{Vol})$ \\
\hline $\mathrm{CO} 2$ & 14,5 & 15,1 & 14,6 & 14,6 & 15,4 & 14,5 & 13,9 & 15,4 & 14 & 14,1 & 15,5 & 15,1 & (\% $\% \mathrm{Vol})$ \\
\hline$H C$ & 8 & 7 & 14 & 12 & 17 & 12 & 16 & 1 & 42 & 18 & 7 & 29 & ( ppmVol) \\
\hline 02 & 0,1 & 0,08 & 0,06 & 0,09 & 0,09 & 0,08 & 0,21 & 0,05 & 0,35 & 0,05 & 0,11 & 0,09 & (\%Vol) \\
\hline Lambda & 1,004 & 1,003 & 1,002 & 1,004 & 1,003 & 1,003 & 1,009 & 1,002 & 1,016 & 1,001 & 1,005 & 1,003 & $(-)$ \\
\hline
\end{tabular}

Autor: Marco Noroña

Luego de las pruebas de laboratorios determinamos mediante los valores arrojados que la bujía que emite menor emisión de gases es la NGK calibrado sus electrodos a 0,85mm a 700 rpm utilizando el filtro de aire original. A continuación, se detallan las tabulaciones de los datos mediante las siguientes gráficas con las diferentes calibraciones de los electrodos de las bujías. 


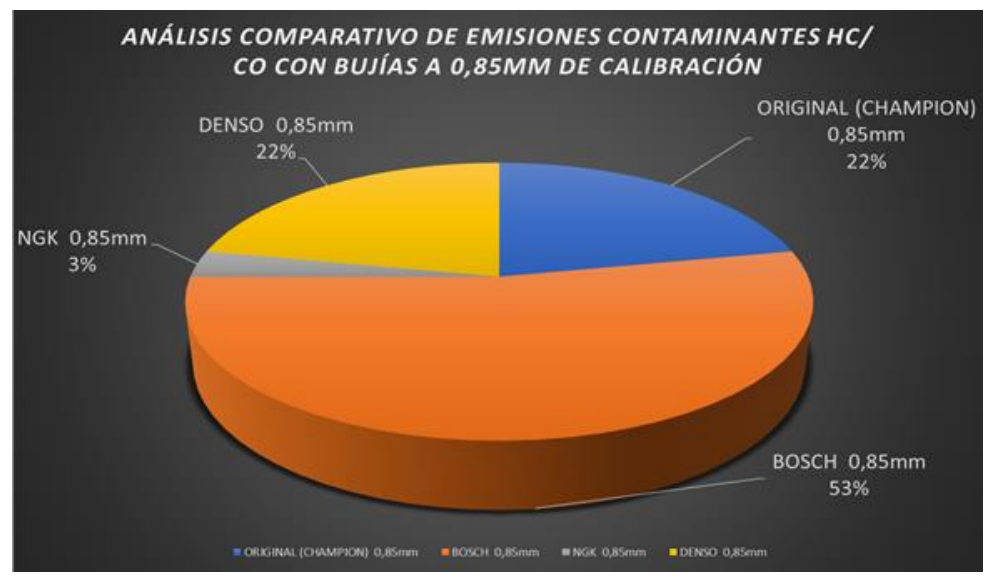

Figura 11. Análisis de Gases a 700rpm combustible 87 octanos variable bujía calibración electrodo a $0.85 \mathrm{~mm}$ con filtro de aire original - Autor: Marco Noroña

Con la información de la primera tabla (tabla 9) donde nos arroja como resultado que la bujía marca NGK es la que emite menor contaminación al ambiente calibrado sus electrodos a $0,85 \mathrm{~mm}$ y a 700rpm ahora en la siguiente tabla (tabla 10) vamos a combinar la bujía marca NGK calibrados sus electrodos a $0,85 \mathrm{~mm}$ con cuatro marcas de filtros de aire diferentes, uno es el original del vehículo y los otros tres son alternos.

Tabla 10. Análisis de resultado de valores de emisiones de gases utilizando la bujía marca BOSCH vs varias variables

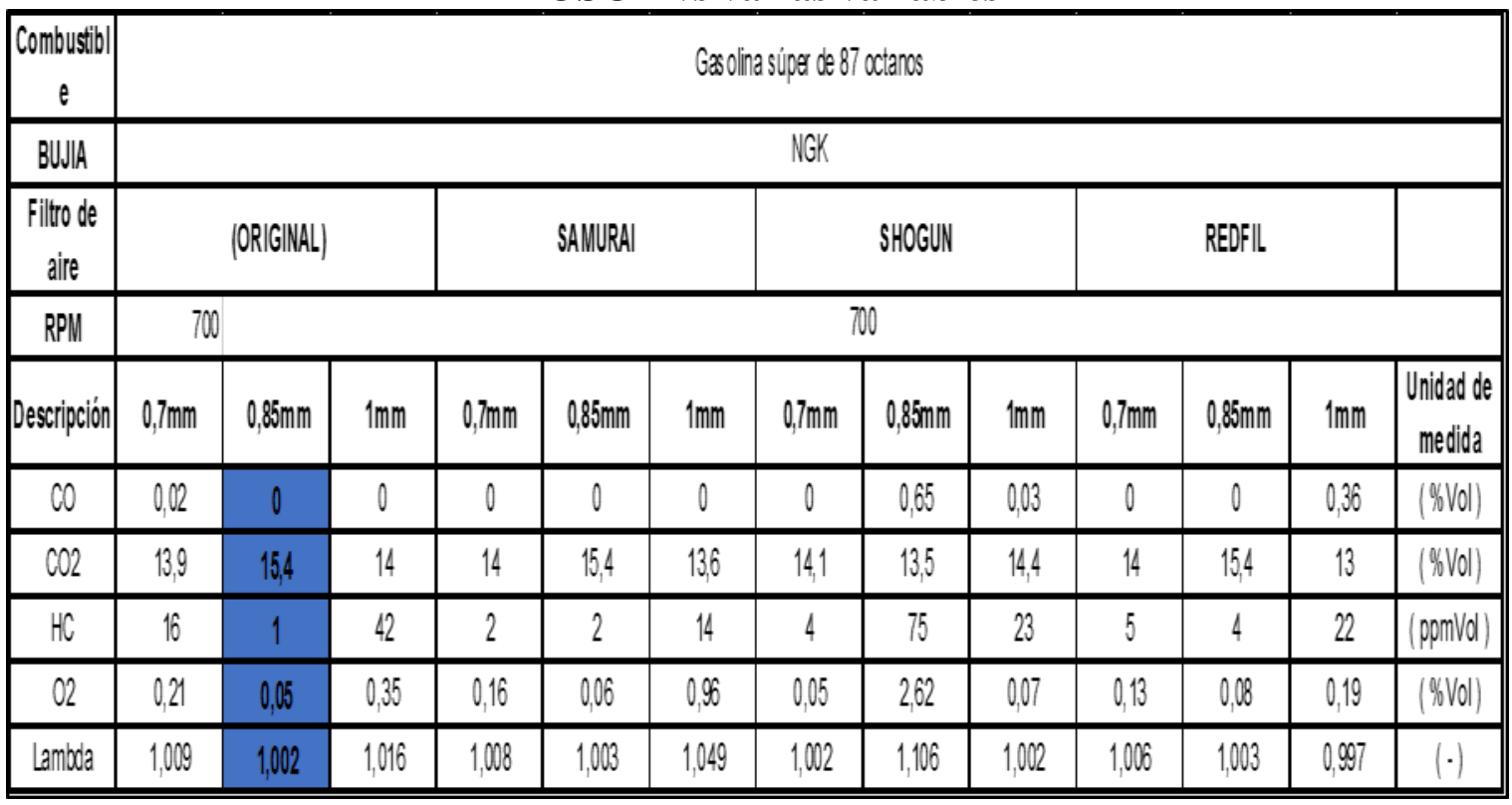

Autor: Marco Noroña

En este caso según las pruebas de laboratorio nos arrojan como resultado que el mismo filtro de aire Original emite menos gases contaminantes junto con la bujía marca NGK calibrados sus electrodos a $0,85 \mathrm{~mm}$ a 700rpm. A continuación, se detallan las tabulaciones de los datos mediante las siguientes gráficas con las diferentes calibraciones de los electrodos de las bujías. 


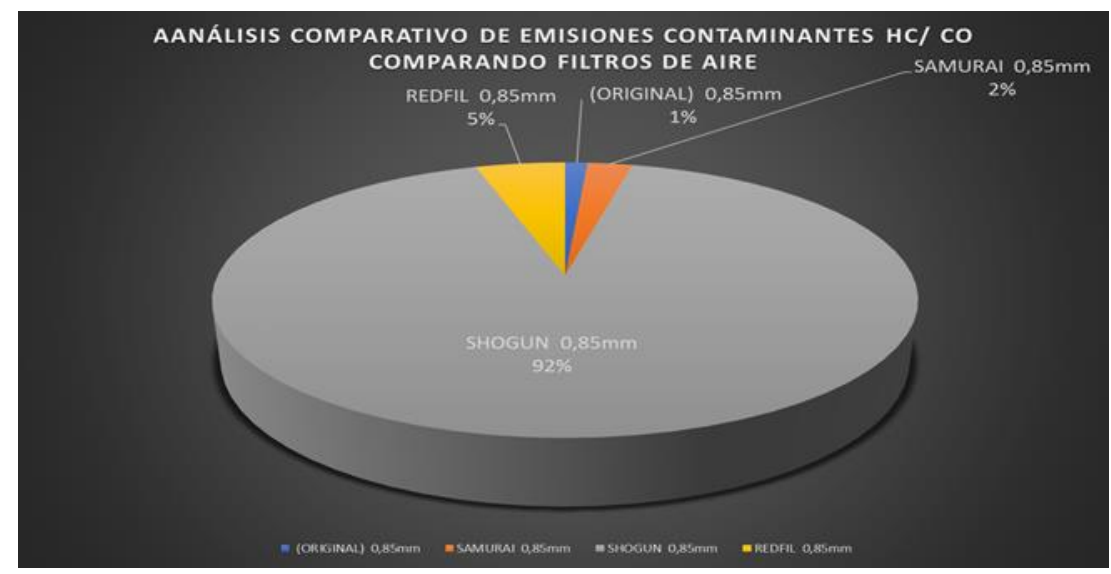

Figura 12. Análisis de Gases a 700rpm combustible 87 octanos variable filtros de aire con bujía NGK electrodo a 0,85mm - Autor: Marco Noroña

\section{Medición De Resultados Utilizando Combustible De 87 Octanos A 2500rpm}

A continuación, detallamos la tabla de valores con los resultados obtenidos luego de hacer las pruebas de laboratorio utilizando el filtro de aire original con las cuatro diferentes marcas de bujías a tres calibraciones de electrodos diferentes a 2500rpm utilizando combustible de 87 octanos. En esta tabla (tabla 11) vamos a obtener como resultado con que bujía y a que calibración del electrodo emite menor emisión de gases el vehículo Chevrolet Sail 1.4.

Tabla 11. Análisis de resultado de valores de emisiones de gases utilizando la bujía marca NGK vs varias variables

\begin{tabular}{|c|c|c|c|c|c|c|c|c|c|c|c|c|c|}
\hline Combusibl & \multicolumn{13}{|c|}{ Gasolina súper de 87 octanos } \\
\hline Filtro de & \multicolumn{13}{|c|}{ ORIGINAL } \\
\hline Bujjas & \multicolumn{3}{|c|}{ ORIGINAL (CHAMPION) } & \multicolumn{3}{|c|}{ BOSCH } & \multicolumn{3}{|c|}{ NGK } & \multicolumn{3}{|c|}{ DENSO } & \\
\hline RPM & \multicolumn{13}{|c|}{2500} \\
\hline Descripción & $0,7 \mathrm{~mm}$ & $0,85 \mathrm{~mm}$ & $1 \mathrm{~mm}$ & $0,7 \mathrm{~mm}$ & $0,85 \mathrm{~mm}$ & $1 \mathrm{~mm}$ & $0,7 \mathrm{~mm}$ & $0,85 \mathrm{~mm}$ & $1 \mathrm{~mm}$ & $0,7 \mathrm{~mm}$ & $0,85 \mathrm{~mm}$ & $1 \mathrm{~mm}$ & dad de med \\
\hline $\mathrm{CO}$ & 0,27 & 0 & 0,04 & 0,03 & 0 & 0,01 & 0,02 & 0 & 0,35 & 0,04 & 0,4 & 0 & $(\% \mathrm{VOl})$ \\
\hline $\mathrm{CO2}$ & 14,3 & 15,1 & 14,5 & 14,5 & 15,4 & 14,4 & 13,1 & 15,4 & 13 & 14,1 & 15,3 & 15 & $(\% \mathrm{VOO})$ \\
\hline$H C$ & 26 & 5 & 14 & 16 & 15 & 25 & 4 & 6 & 59 & 40 & 110 & 17 & (ppmVol) \\
\hline 02 & 0,09 & 0,14 & 0,16 & 0,06 & 0,09 & 0,16 & 0,36 & 0,08 & 0,37 & 0,08 & 0,07 & 0,09 & $(\% \mathrm{VOl})$ \\
\hline Lambda & 0,995 & 1,006 & 1,006 & 1,001 & 1,004 & 1,006 & 1,018 & 1,003 & 1,005 & 1,001 & 0,988 & 1,004 & $(-)$ \\
\hline
\end{tabular}

Autor: Marco Noroña

Luego de las pruebas de laboratorios determinamos mediante los valores arrojados que la bujía que emite menor emisión de gases es la NGK calibrado sus electrodos a 0,7mm a 2500 rpm utilizando el filtro de aire original. A continuación, se detallan las tabulaciones de los datos mediante las siguientes gráficas con las diferentes calibraciones de los electrodos de las bujías. 


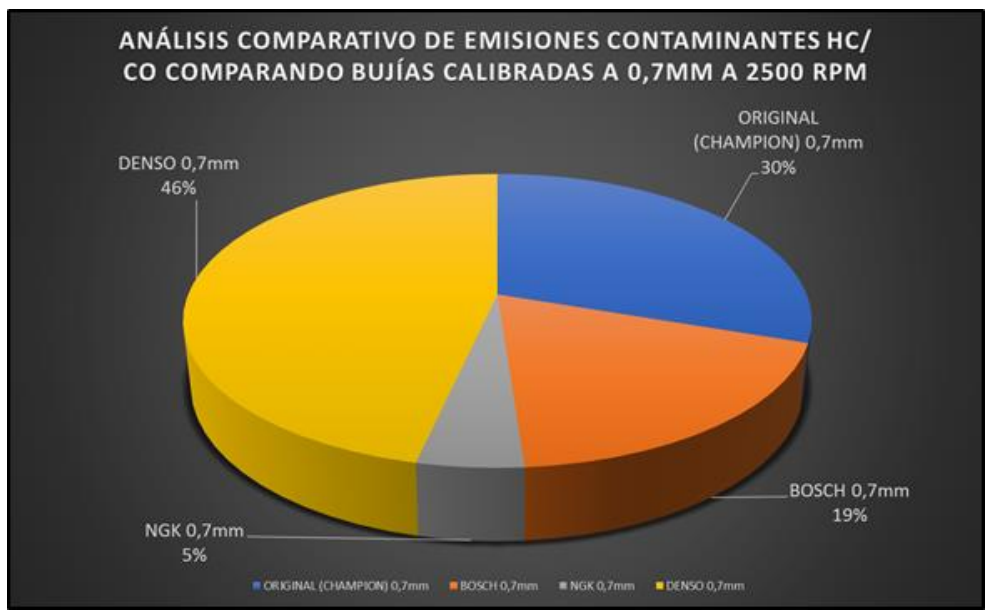

Figura 13. Análisis de Gases a 2500rpm combustible 87 octanos variable bujía calibración electrodo a $0.7 \mathrm{~m}$ con filtro de aire original - Autor: Marco Noroña

Con la información de la primera tabla (tabla 11) donde nos arroja como resultado que la bujía marca NGK es la que emite menor contaminación al ambiente calibrado sus electrodos a $0,85 \mathrm{~mm}$ y a $2500 \mathrm{rpm}$ ahora en la siguiente tabla (tabla 12 ) vamos a combinar la bujía marca NGK calibrados sus electrodos a $0,85 \mathrm{~mm}$ con cuatro marcas de filtros de aire diferentes, uno es el original del vehículo y los otros tres son alternos.

Tabla 12. Análisis de resultado de valores de emisiones de gases utilizando la bujía marca NGK vs varias variables

\begin{tabular}{|c|c|c|c|c|c|c|c|c|c|c|c|c|c|}
\hline Combustibl & \multicolumn{13}{|c|}{ Gasolina súver de 87 octanos } \\
\hline BUJAA & \multicolumn{13}{|c|}{ NGK } \\
\hline \begin{tabular}{|c|} 
Filtro de \\
aire
\end{tabular} & \multicolumn{3}{|c|}{ SHANGAI (ORIGINAL) } & \multicolumn{3}{|c|}{ SAMURAI } & \multicolumn{3}{|c|}{ SHOGUN } & \multicolumn{3}{|c|}{ REDFIL } & \\
\hline RPM & \multicolumn{13}{|c|}{2500} \\
\hline Descripción & $0,7 \mathrm{~mm}$ & $0,85 \mathrm{~mm}$ & $1 \mathrm{~mm}$ & $0,7 \mathrm{~mm}$ & $0,85 \mathrm{~mm}$ & $1 \mathrm{~mm}$ & $0,7 \mathrm{~mm}$ & $0,85 \mathrm{~mm}$ & $1 \mathrm{~mm}$ & $0,7 \mathrm{~mm}$ & $0,85 \mathrm{~mm}$ & $1 \mathrm{~mm}$ & $\begin{array}{c}\text { Unidad de } \\
\text { medida }\end{array}$ \\
\hline $\mathrm{CO}$ & 0,02 & 0 & 0,35 & 0 & 0 & 0 & 0,01 & 0,03 & 0,01 & 0,02 & 0 & 0,35 & (\%VOl) \\
\hline $\mathrm{CO} 2$ & 13,1 & 15,4 & 13 & 14 & 15,4 & 14,2 & 14 & 15,2 & 14,4 & 13,1 & 15,4 & 13 & (\%VOl) \\
\hline $\mathrm{HC}$ & 4 & 6 & 59 & 7 & 2 & 11 & 8 & 25 & 23 & 4 & 6 & 59 & (ppmVol) \\
\hline 02 & 0,36 & 0,08 & 0,37 & 0,08 & 0,07 & 0,08 & 0,07 & 0,15 & 0,09 & 0,36 & 0,08 & 0,37 & (\%VOl) \\
\hline Lambda & 1,018 & 1,003 & 1,005 & 1,004 & 1,003 & 1,003 & 1,002 & 1,005 & 1,003 & 1,018 & 1,003 & 1,005 & $(\cdot)$ \\
\hline
\end{tabular}

Autor: Marco Noroña

Luego de las pruebas de laboratorios determinamos mediante los valores arrojados que la bujía que emite menor emisión de gases es la NGK calibrado sus electrodos a 0,85mm a 2500 rpm utilizando el filtro de aire SAMURAI. A continuación, se detallan las tabulaciones de los datos mediante las siguientes gráficas con las diferentes calibraciones de los electrodos de las bujías. 


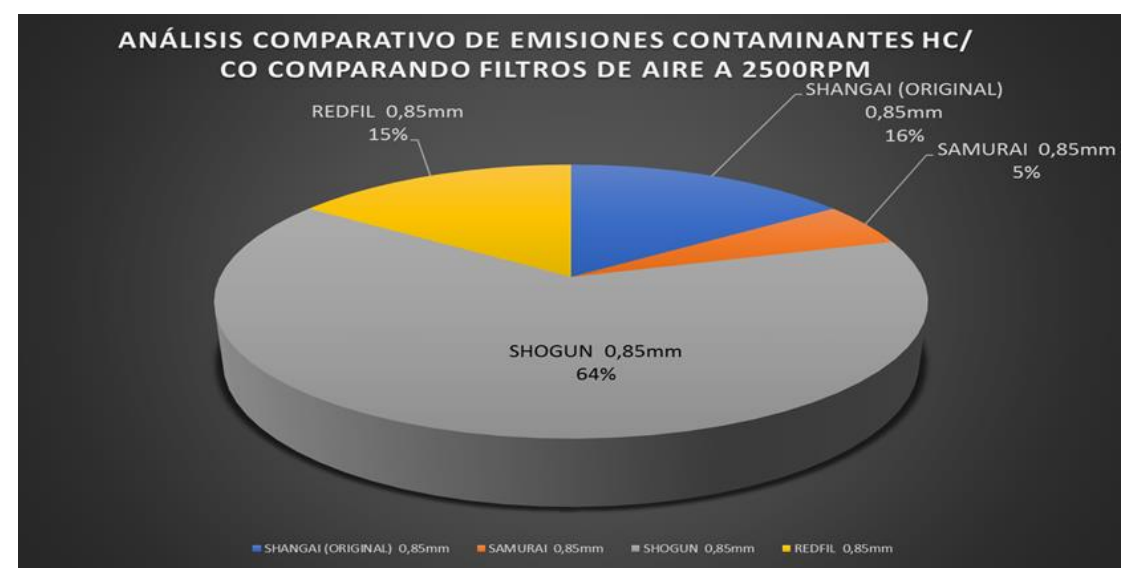

Figura 14. Análisis de Gases a 2500rpm combustible 87 octanos variable filtros de aire con bujía NGK electrodo a $0,7 \mathrm{~mm}$

Autor: Marco Noroña

\section{Conclusiones y Recomendaciones}

\section{Conclusiones}

En base a los resultados obtenidos de las tabulaciones realizadas tanto a ralentí como a $2500 \mathrm{rpm}$ y considerando las combinaciones de filtros con bujías se recomienda utilizar lo siguiente elementos en el vehículo Chevrolet Sail 1.4. Para tener la menor cantidad de emisiones de gases contaminantes cuando su combustible sea de 92 octanos:

- Bujías marca BOSCH

- Calibración de los electrodos de las bujías a $1 \mathrm{~mm}$.

- Filtro de aire Original.

Cuando dicho vehículo utilice un combustible de 82 octanos se recomienda utilizar en cambio bujías NGK y ya sea filtro original o a su vez el filtro SAMURAI.

Estas conclusiones las podemos resumir en el siguiente cuadro:

\begin{tabular}{lllll}
\hline Repuesto & \multicolumn{2}{l}{ Combustible De 92 Octanos } & \multicolumn{2}{l}{ Combustible De 82 Octanos } \\
\cline { 2 - 5 } & Ralentí & 2500 Rpm & Ralentí & 2500 Rpm \\
Bujías & Bosch & Bosch & Ngk & Ngk \\
Filtro De Aire & Samurai & Original & Original & Samurái / Original \\
\hline
\end{tabular}

\section{Recomendaciones}

En base a los resultados obtenidos y a las conclusiones emitidas de acuerdo con las tabulaciones realizadas tanto a $700 \mathrm{rpm}$ y $2500 \mathrm{rpm}$ y considerando las combinaciones de filtros y bujías se recomienda utilizar lo siguiente elementos en el vehículo Chevrolet Sail 1.4. Para tener la menor cantidad de emisiones de gases contaminantes: 
- Bujías marca BOSCH

- Calibración de los electrodos de las bujías a $1 \mathrm{~mm}$.

- $\quad$ Filtro de aire ORIGINAL.

\section{Bibliografía}

Autor Libro Técnica en la comprobación “Comprobación de los gases de escape con los aparatos de medida Bosch"

Presentación de Curso Técnico del Sail.

Electrónica y Electricidad Automotriz Volumen 2.

Manual Técnico Fuel Inyection Autor Jesús Rueda Santander.

Técnica de gases de escape para motores de gasolina.

Electrónica y Electricidad Automotriz Volumen 1.

Manual del Automóvil Reparación y Mantenimiento.

Curso Técnico del Sail.

Stuttgar G. (1980). Tecnología del automóvil). Tomo 2

Alonso, J. (2003). Técnicas del automóvil. Inyección de gasolina y dispositivos anticontaminantes. Thompson. 2da. Edición. Madrid

Alonso, J. (2004). Tecnologías avanzadas del automóvil. Thompson. 4ta. Edición. Madrid

Santander, J. (2005). Técnica mecánica y electrónica automotriz. Diseli. Tomo 1,2y 3. Colombia

Castro, M. (2001). Inyección de gasolina. Edición Ceac

Manual del automóvil reparación y mantenimiento. Edita Cultura S.A..Edición 2001. Madrid

Electrónica y electricidad automotriz. México Digital Comunicación S.A. Volumen 1 y 2. México

Rueda, J. (2005). Manual técnico de fuellinjection. Diseli. Tomo 1. Colombia

Norbye J. (2004). Manual de sistemas de fuel injection. Prentice Hall

CEAC. (2003). Manual del automóvil. Editorial CEAC

Martínez, G. (2001). Manual del automóvil: el motor de gasolina. Tomo 1 
Martínez, G. Manual práctico del automóvil. Editorial Cultural S.A.

http://www.cise.com/portal/notas-tecnicas/item/302-an\%C3\%A1lisis-de-los-gases-de-escape-delos-motores-de-combusti\%C3\%B3n-interna.html

http://www.banrepcultural.org/node/92123

http://www.globaltech-car.com/detalle.php?idprd=79

http://aguilaslibres.superforos.org/t285-bujias-concepto-de-funcionamiento-y-nomenclatura-ngk http://es.wikipedia.org/wiki/Convertidor_catal\%C3\%ADtico

http://www.ocio.net/estilo-de-vida/ecologismo/lluvia-acida/

http://www.aficionadosalamecanica.net/ventilacion-positiva-carter.htm

WwW.naikontuning.com

Biblioteca virtual Luis Ángel Arango

http://e-auto.com.mx/manual_detalle.php?manual_id=2 